\title{
Electrochemical nanostructuring of (111) oriented GaAs crystals: from porous structures to nanowires
}

\author{
Elena I. Monaico ${ }^{1}$, Eduard V. Monaico ${ }^{*}$, Veaceslav V. Ursaki ${ }^{1,2}$, Shashank Honnali ${ }^{3}$, \\ Vitalie Postolache ${ }^{1}$, Karin Leistner ${ }^{3}$, Kornelius Nielsch ${ }^{3}$ and Ion M. Tiginyanu ${ }^{1,2}$
}

\section{Full Research Paper}

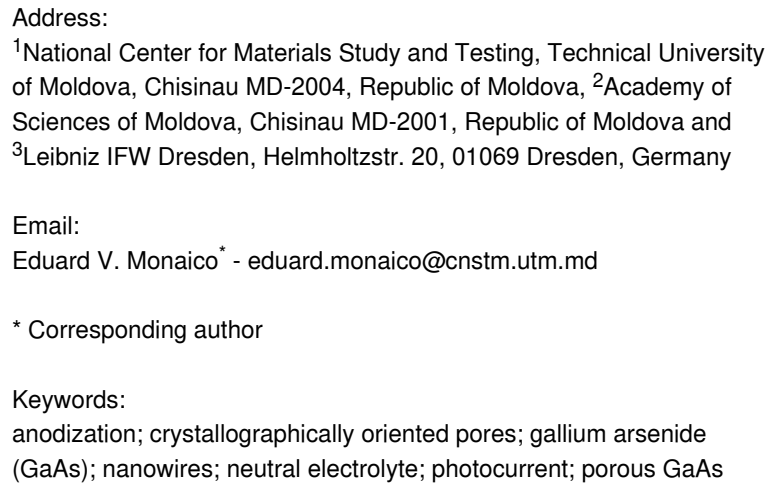

${ }^{1}$ National Center for Materials Study and Testing, Technical University of Moldova, Chisinau MD-2004, Republic of Moldova, ${ }^{2}$ Academy of Sciences of Moldova, Chisinau MD-2001, Republic of Moldova and ${ }^{3}$ Leibniz IFW Dresden, Helmholtzstr. 20, 01069 Dresden, Germany

Beilstein J. Nanotechnol. 2020, 11, 966-975.

doi:10.3762/bjnano.11.81

Received: 29 March 2020

Accepted: 27 May 2020

Published: 29 June 2020

This article is part of the thematic issue "Functional nanostructures for electronics, spintronics and sensors".

Guest Editor: A. S. Sidorenko

(C) 2020 Monaico et al.; licensee Beilstein-Institut. License and terms: see end of document.

\begin{abstract}
A comparative study of the anodization processes occurring at the GaAs(111)A and GaAs(111)B surfaces exposed to electrochemical etching in neutral $\mathrm{NaCl}$ and acidic $\mathrm{HNO}_{3}$ aqueous electrolytes is performed in galvanostatic and potentiostatic anodization modes. Anodization in $\mathrm{NaCl}$ electrolytes was found to result in the formation of porous structures with porosity controlled either by current under the galvanostatic anodization, or by the potential under the potentiostatic anodization. Possibilities to produce multilayer porous structures are demonstrated. At the same time, one-step anodization in a $\mathrm{HNO}_{3}$ electrolyte is shown to lead to the formation of GaAs triangular shape nanowires with high aspect ratio (400 nm in diameter and $100 \mu \mathrm{m}$ in length). The new data are compared to those previously obtained through anodizing GaAs(100) wafers in alkaline KOH electrolyte. An IR photodetector based on the GaAs nanowires is demonstrated.
\end{abstract}

\section{Introduction}

Electrochemical technology became an established and costeffective approach for the preparation of porous semiconductor matrices and arrays of nanowires with tailored architecture at the submicrometer scale [1-3]. Semiconductor nanotemplates provide many possibilities for nanofabrication through electrochemically filling the pores with metallic nanostructures such as nanowires or nanotubes, resulting in the production of $2 \mathrm{D}$ metallo-semiconductor interpenetrating networks, which are promising for various nanoelectronic, optoelectronic, plasmonic, and nanophotonic applications [4-6]. While the growth of crystallographically oriented and current line oriented pores has been demonstrated in a variety of semiconductors [1-3], to date, only crystallographically oriented pores were observed in GaAs crystalline wafers. This observation is a factor limiting 
the possibilities for the preparation of various GaAs nanostructures, including nanowires.

Semiconductor nanowires, particularly III-V compound nanowires, show potential for their use as active components in solar cells [7-10], photodetectors [11], light-emitting diodes [12] transistors [13], and other applications. A uniform array of parallel nanowires with diameters of about $50 \mathrm{~nm}$ and oriented normally to a InP wafer, i.e., along the crystallographic [100] orientation, was obtained after anodic etching at elevated applied voltages [14]. High-aspect-ratio GaAs pillar arrays with triangular cross section were prepared by combining colloidal crystal templating, anisotropic chemical etching, localized anodic etching, and isotropic anodic oxidation $[15,16]$. However, this is a complex multistep technology. A more simple and cost-effective technology was applied for obtaining triangular GaAs nanowires through electrochemical etching of GaAs(100) surfaces in aqueous $\mathrm{KOH}$ solution [17]. However, this process was difficult to control. The bundles of GaAs nanowires were formed only in some regions of the surface and the orientation of the arrays was basically random.

Usually, acidic or alcaline electrolytes are used for the electrochemical preparation of porous semiconductors although the anodization in environmentally friendly electrolytes, including aqueous solutions of $\mathrm{NaCl}$, has attracted increasing attention during the last decade [5,18-21].

In this paper, we report on the electrochemical porosification of GaAs(111) wafers in neutral $\mathrm{NaCl}$ and acidic $\mathrm{HNO}_{3}$ electrolytes, and on optimized technological conditions for the one-step formation of GaAs nanowires with triangular shape and well oriented perpendicularly to the substrate.

\section{Results and Discussion}

In order to study the influence of the applied anodization conditions on the porosification process of (111) oriented GaAs crystals, three different current densities and three different voltages were used for galvanostatic and potentiostatic modes, respectively. SEM images of a GaAs crystal anodized on both surfaces in $1.75 \mathrm{M} \mathrm{NaCl}$ electrolyte are given in Figure 1. The porous features produced by $\mathrm{GaAs}$ anodization in $\mathrm{NaCl}$ electrolyte are similar to those previously observed in GaAs samples with the same carrier concentration anodized in $\mathrm{HCl}$ electrolyte [1]. This observation corroborates the results obtained from other III-V semiconductors, which state that the etching behavior depends mainly on the anions rather than the cations [22], because the anions of $\mathrm{NaCl}$ and $\mathrm{HCl}$ electrolytes are the same. The morphology of the porous structure produced by galvanostatic anodization of GaAs(111)A surface (Figure 1A) consists of two sets of $\langle 111\rangle$ crystallographically oriented pores intersecting each other [2]. According to previous studies, the main property of crystallographically oriented pores is their growth along definite crystallographic directions. On substrates with sphalerite crystal structures, the pores grow along the $\langle 111\rangle$ B crystallographic directions, regardless of the initial surface orientation, with an angle of approximately $109^{\circ}$ between the pores. The pores tend to have a triangular cross section while the pore walls and tips exhibit a pronounced crystallographic anisotropy. A specific characteristic feature of crystallographically oriented pores is their ability to intersect each other without changing their direction of propagation during growth.
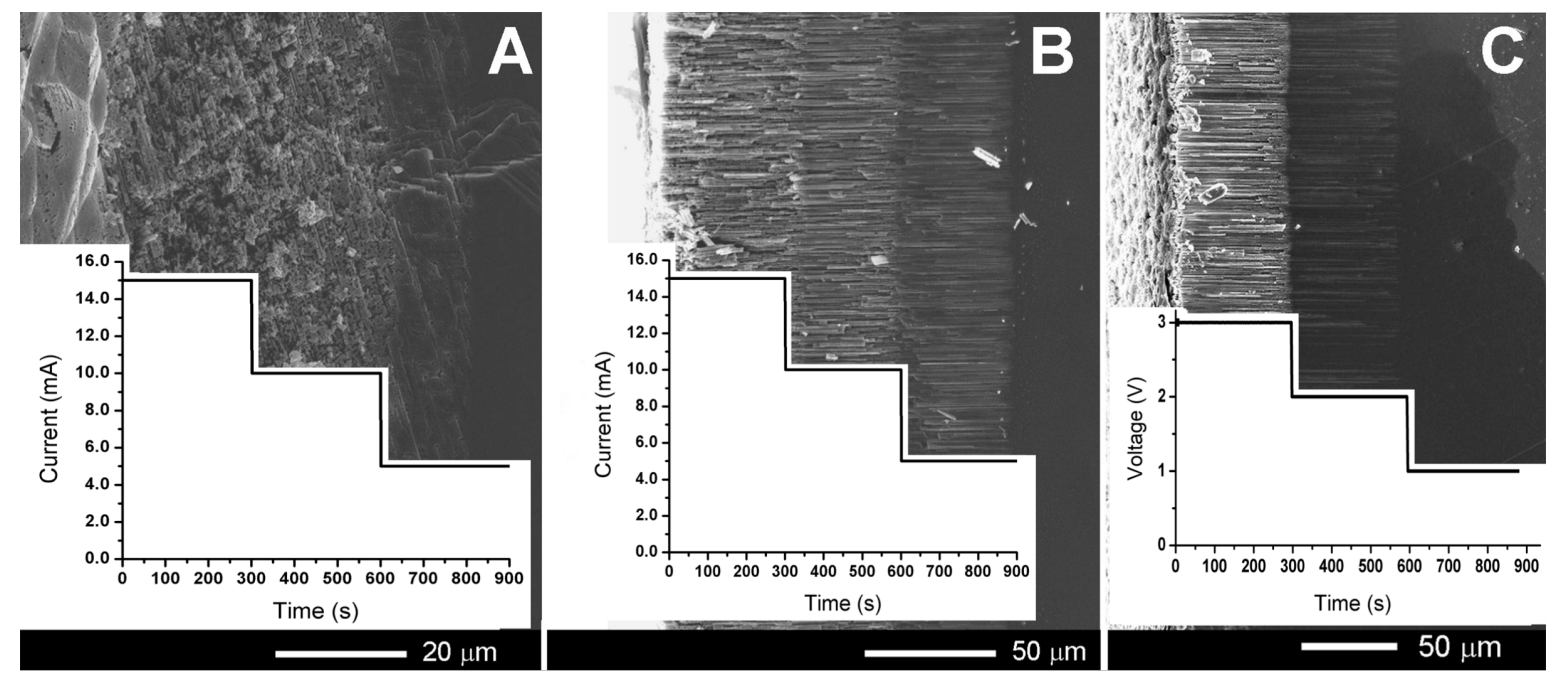

Figure 1: SEM images in cross section of porous GaAs layers for three different conditions of anodization in $1.75 \mathrm{M} \mathrm{NaCl:} \mathrm{(A)} \mathrm{galvanostatic} \mathrm{regime} \mathrm{on}$ the $\mathrm{GaAs}(111) \mathrm{A}$ surface; (B) galvanostatic regime on the $\mathrm{GaAs}(111) \mathrm{B}$ surface; (C) potentiostatic regime on the GaAs(111)B surface. The substrate is on the right side of the images. The inserted plots represent the applied currents $(A, B)$ and the applied potential $(C)$ during anodization. 
As one can see from Figure $1 \mathrm{~A}$, the degree of porosity decreases with decreasing the anodization current density.

The situation is different when the GaAs crystals are anodized on the (111)B surface. During anodization in the galvanostatic mode at current densities similar to those applied during anodizing the (111)A surface $(15,10$, and $5 \mathrm{~mA})$, three porous layers are formed with different degrees of porosity, but the pores are parallel to each other and they grow perpendicularly to the crystal surface (Figure 1B). The same propagation of pores was observed for the potentiostatic mode of anodization (Figure 1C). Switching the anodization voltage to $1 \mathrm{~V}$ stops the formation of the porous layer, since this value is below the pore nucleation potential $(1.8 \mathrm{~V})$ established from the $I-V$ characteristic, which is in agreement with the doping level of the used crystal. We will focus further on the results obtained by anodization of the (111)B surface because the resulting morphology is of great interest for the development of porous nanotemplates and elaboration of free-standing nanomembranes based on GaAs.

The results of a comparative anodization study of the GaAs(111)B surface in galvanostatic and potentiostatic regimes are illustrated in the SEM images of Figure 2. Along with pores
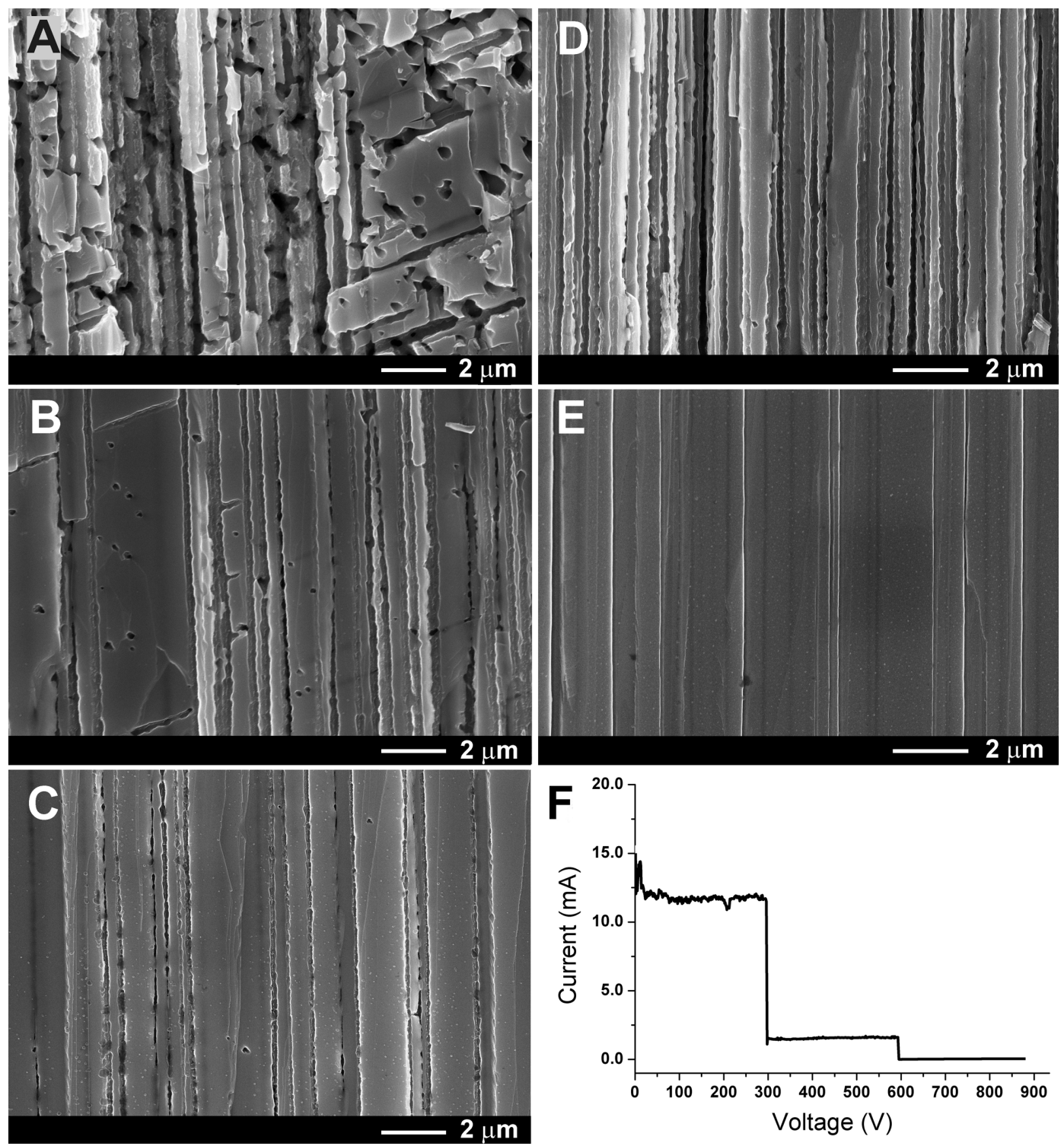

Figure 2: $(A-C)$ SEM images in cross section at higher magnification of the porous layers obtained by anodization of the GaAs(111)B surface in galvanostatic regime, corresponding to the images illustrated in Figure 1B. (D, E) SEM images of samples anodized in potentiostatic regime, corresponding to images illustrated in Figure 1C. (F) Evolution of the current during anodization in potentiostatic regime of the GaAs(111)B surface at applied voltages of 3,2 and $1 \mathrm{~V}$. 
propagating perpendicularly to the substrate surface, pores growing in other directions are observed in the cross-sectional view (Figure 2A), hinting at the formation of pores that are tilted and intersect each other. However, with the decrease of the current density (Figure 2B,C), the number of tilted pores is drastically reduced, and pores oriented perpendicularly to the surface predominate in the produced porous layer (Figure 2C). The morphology of the layer produced by anodization in the potentiostatic regime presented in Figure 2D,E looks more uniform (note that the pores grow perpendicularly to the surface). It should be mentioned, however, that the pore walls are not smooth, which is observed for both potentiostatic and galvanostatic anodization regimes. This can be explained by analyzing the current as a function of the time at constant voltage (Figure $2 \mathrm{~F}$ ). At the applied potential of $3 \mathrm{~V}$, the registered current is practically stable at a value of $13 \mathrm{~mA}$, but some fluctuations in the current can be easily observed. Reducing the anodization voltage to $2 \mathrm{~V}$ leads to a decrease of the current down to $5 \mathrm{~mA}$. This value remains quite stable. So, by anodizing the sample in the potentiostatic mode at relatively low voltage, which does not introduce self-oscillations in the registered current, it is possible to obtain pores with smooth walls (Figure 2E).

According to Li and co-workers [17], the anodization of GaAs with a carrier concentration of about $10^{18} \mathrm{~cm}^{-3}$ in a $\mathrm{KOH}$ electrolyte can be categorized into three etching modes, deduced from the analysis of the current-voltage plot at a scan rate of $5 \mathrm{mV} \cdot \mathrm{s}^{-1}$. Practically no etching occurs when the applied voltage is below $3 \mathrm{~V}$, which is the pore formation potential. The first etching mode is characterized by the formation of triangular nanowires, which occurs at an applied potential in the range of 3.0-4.5 V. The second mode corresponds to surface texturing occurring at an applied potential in the range of 4.5-6.5 V. At an applied potential higher than $6.5 \mathrm{~V}$ the sample is electropolished. Taking these observations into account, we increased the applied potential in our experiments to $4 \mathrm{~V}$, with the purpose of producing GaAs nanowires. However, the analysis of SEM images after anodization in $\mathrm{NaCl}$ electrolyte with an applied potential of $4 \mathrm{~V}$ revealed that no nanowires are formed. Instead a higher number of tilted pores was formed, similar to the morphology produced under anodization in the galvanostatic regime with a current of $14 \mathrm{~mA}$ (Figure 2A). Moreover, an increase of the applied potential to $6 \mathrm{~V}$ leads to the formation of $\mathrm{GaAs}$ nanowires, but the number of tilted pores that intersect the vertical nanowires increases, resulting in the formation of interrupted nanowires (Figure 3A). A further increase of the applied voltage is not recommended, because the formed nanowires are destroyed. We are interested in the fabrication of pores strictly perpendicular to the crystal surface, which would enable us to fabricate stable nanowires.
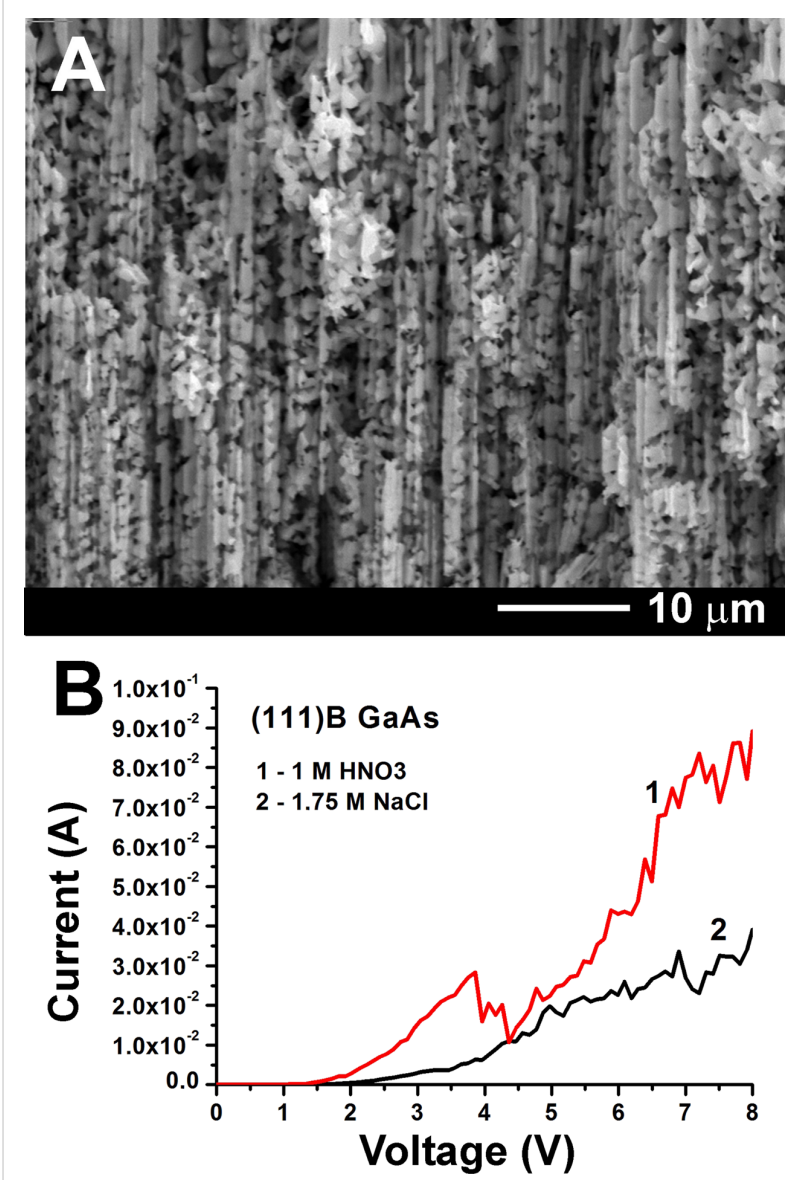

Figure 3: (A) SEM images of the formation of interrupted GaAs nanowires on the (111)B surface anodized in $\mathrm{NaCl}$ electrolyte at $6 \mathrm{~V}$. (B) The polarization curves measured at a scan rate of $50 \mathrm{mV} \cdot \mathrm{s}^{-1}$ at the beginning of the anodization process of a $\mathrm{GaAs}(111) \mathrm{B}$ crystal in $1 \mathrm{M} \mathrm{HNO}_{3}$ (curve 1) and $1.75 \mathrm{M} \mathrm{NaCl}$ (curve 2).

One can conclude that anodizations in $\mathrm{NaCl}$ electrolyte and in aqueous $\mathrm{HCl}$ solution are suitable for the production of porous layers with various morphologies, rather than for the fabrication of GaAs nanowires. Since, on one hand, nanowires were produced through anodization in alkaline $\mathrm{KOH}$ electrolyte [17] and, on the other hand, the etching behavior in acidic electrolytes is mainly determined by the anions [22], we tried to obtain GaAs nanowires through anodization in acidic electrolyte with another type of anion. A comparison of the polarization curves measured at the beginning of the anodization process of a GaAs(111)B crystal in $1 \mathrm{M} \mathrm{HNO}_{3}$ or $1.75 \mathrm{M} \mathrm{NaCl}$ electrolyte is presented in Figure 3B. It can be observed that current oscillations occur at applied potentials higher than $4 \mathrm{~V}$ for both electrolytes. We suppose that these self-induced oscillations are related to the simultaneous formation of pores along the $\langle 111\rangle \mathrm{B}$ directions and an increasing contribution of tilted pores. At high anodizing voltages electropolishing occurs in both electrolytes. The potential for initiating electropolishing is $4.5 \mathrm{~V}$ and $6 \mathrm{~V}$ for anodization in $\mathrm{HNO}_{3}$ and $\mathrm{NaCl}$ electrolyte, 
respectively. Note that the value for anodizing in $\mathrm{NaCl}$ electrolyte agrees with previously reported data [17].

Arrays of GaAs nanowires with a very high aspect ratio and well oriented perpendicularly to the (111)B crystal surface were produced under anodization at an applied potential of $3 \mathrm{~V}$ for 20 min in $1 \mathrm{M} \mathrm{HNO}_{3}$ electrolyte (Figure 4A). The obtained triangular nanowires with a diameter of $400 \mathrm{~nm}$ are $100 \mu \mathrm{m}$ in length, i.e., the aspect ratio is around 250. The uniform distribution of the nanowires across the sample surface is illustrated in Figure 4C. Interestingly, the anodization potential for producing nanowires in the acidic $1 \mathrm{M} \mathrm{HNO}_{3}$ electrolyte is similar to that required for producing GaAs nanowires in alkaline 5\% $\mathrm{KOH}$ electrolyte [17].

Thus, triangular-shape GaAs nanowires with a diameter ranging from 200 to $400 \mathrm{~nm}$ can be produced by electrochemical etching of $\mathrm{GaAs}(100)$ wafers with a carrier concentration of the order of $10^{18} \mathrm{~cm}^{-3}$ in $\mathrm{KOH}$ electrolyte, or by etching of GaAs(111)B substrates in $\mathrm{HNO}_{3}$ electrolyte. However, the bundles of GaAs nanowires are formed only in some regions of the surface anodized in $\mathrm{KOH}$ electrolyte, and the orientation of the arrays is basically random, while the nanowires produced in $\mathrm{HNO}_{3}$ electrolyte are highly oriented perpendicularly to the wafer surface. No nanowires were reported after GaAs anodization in $\mathrm{H}_{2} \mathrm{SO}_{4}$ electrolyte, which was used for uniform pore nucleation in a two-step anodization process and the preparation of porous structures consisting of crossing pores [1,23]. GaAs nanocolumns with a diameter of about $200 \mathrm{~nm}$ were obtained previously through anodization of $\mathrm{GaAs}(100)$ substrates in aqueous $\mathrm{HCl}$ solution [24], but the nanocolumns were penetrated by a large number of tilted pores. One can conclude that etching in $\mathrm{HNO}_{3}$ electrolyte suppresses the nucleation of tilted pores as compared to anodization in $\mathrm{HCl}$ or $\mathrm{H}_{2} \mathrm{SO}_{4}$ electrolyte, resulting in the fabrication of non-porous GaAs nanowires, which are better suitable for photodetector applications. Highaspect-ratio arrays of spatially ordered triangular GaAs nanopillars with diameters of about $1.5 \mu \mathrm{m}$ were previously fabricated by a combination of electrochemical etching in $\mathrm{HCl}$ electrolyte of a pre-etched GaAs(111) substrate and anisotropic chemical etching $[15,16]$. However, this is a multistep process requiring the use of photolithography with colloidal crystal templating in the first pre-etching step. Fabrication of GaAs nanopillars by metal-assisted chemical etching (MacEtch) also requires lithographic methods for catalytic metal patterning [25,26]. Among non-lithographic methods based on electrochemical etching, to the best of our knowledge, there is only one report on the preparation of high-aspect-ratio vertically aligned GaAs nanowires with a diameter of about $200 \mathrm{~nm}$ and a length of $100 \mu \mathrm{m}$ on $\mathrm{GaAs}(111) \mathrm{B}$ wafers with a carrier concentration in the range of $(1-2) \times 10^{18} \mathrm{~cm}^{-3}$ [27]. Bundles of these nanowires were tested
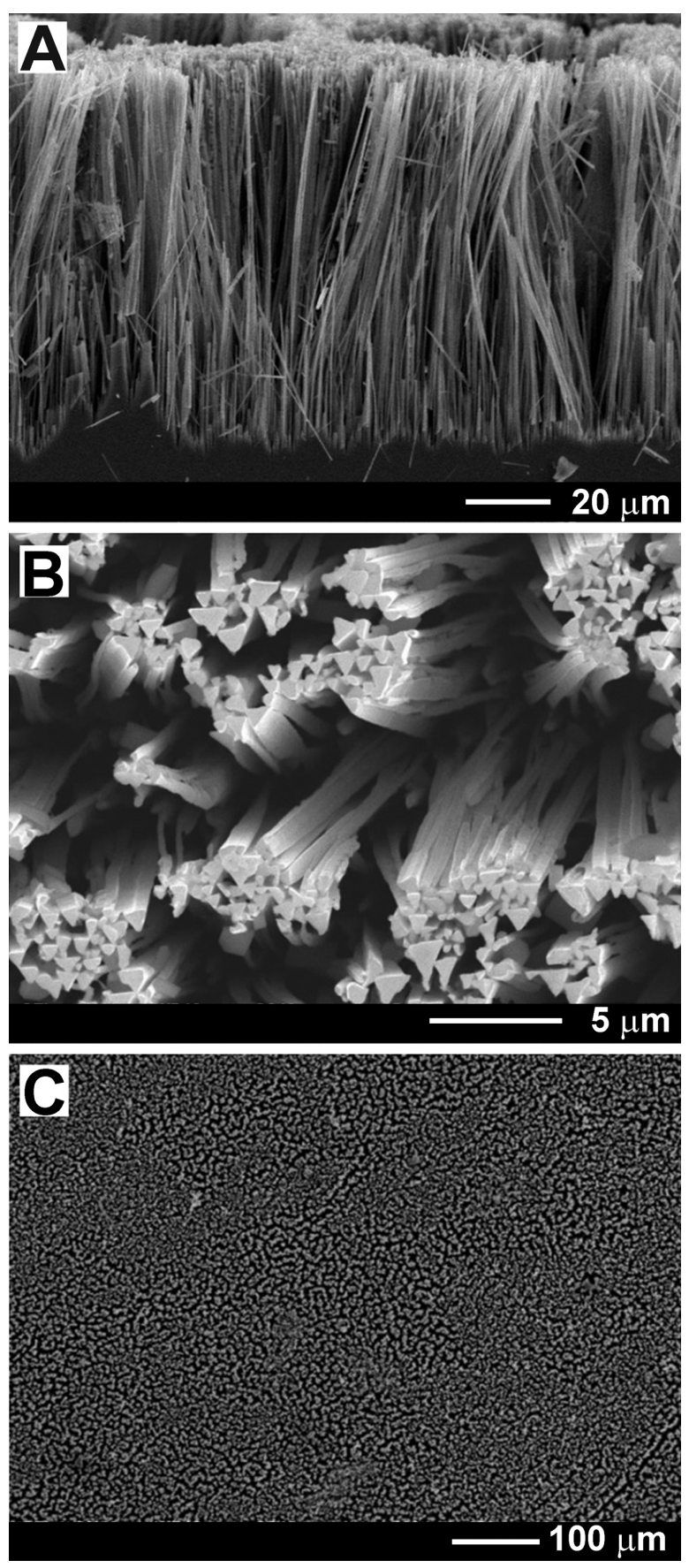

Figure 4: (A) SEM image in cross section of a GaAs(111)B sample anodized at $3 \mathrm{~V}$ for 20 min in $1 \mathrm{M} \mathrm{HNO}_{3}$. (B, C) Top-view SEM images.

for field emission, but not for photodetector applications. The nanowires were produced using electrochemical etching under optimized conditions in a mixed $\mathrm{H}_{3} \mathrm{PO}_{4} / \mathrm{HCl}$ electrolyte followed by chemical etching for reducing the diameter down to $150 \mathrm{~nm}$. The authors of the work did not discuss the diameter distribution of nanowires produced by the process, effectively utilizing a structural feature of spontaneously generated 
patterns. According to the presented scanning electron microscope images, however, there is a relatively broad distribution.

The nanowires produced in the present work also exhibit a diameter distribution with a certain width. However, we believe that the distribution could be narrowed by further optimization of the technological processes. Note that the diameter of a particular nanowire is determined by the width of the space charge region (SCR) set up within the nanopore wall at the interface with the electrolyte during anodization [23], and it equals ca. $2 \times$ SCR [17]. As a result, for higher carrier concentrations in the GaAs wafer thinner nanowires are obtained. The density of the nanowires is quite homogeneous on a large scale (Figure 4C), while the nanowires tend to form bundles at the micrometer scale, similar to the results presented in [27].

Figure 5 compares the photoluminescence (PL) spectra of the as-grown and anodized GaAs samples measured at low temperature. One can see that the shapes of the spectra are similar. The only difference is the emission intensity, which is higher by a factor of about two in the anodized GaAs sample. This means that the PL spectra can be attributed to identical recombination channels. In both samples the PL is dominated by an emission band around $1.32 \mathrm{eV}$ with a weaker PL band at $1.485 \mathrm{eV}$. The $\mathrm{PL}$ band at $1.32 \mathrm{eV}$ is usually attributed to $\mathrm{Si}$ impurities at the $\mathrm{Ga}$ sites forming different complexes, such as $\left(\mathrm{Si}_{\mathrm{Ga}} \mathrm{V}_{\mathrm{Ga}}\right)$

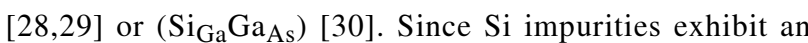
amphoteric behavior in GaAs, they give rise to an acceptor $\mathrm{Si}_{\mathrm{As}}$ state in addition to the $\mathrm{Si}_{\mathrm{Ga}}$ shallow donor state. The second PL band at $1.485 \mathrm{eV}$ is related to the recombination of electrons from the conduction band with holes trapped by the $\mathrm{Si}_{\mathrm{As}}$ state the energy level of which is situated $35 \mathrm{meV}$ above the valence band [31]. The higher intensity of the emission from the

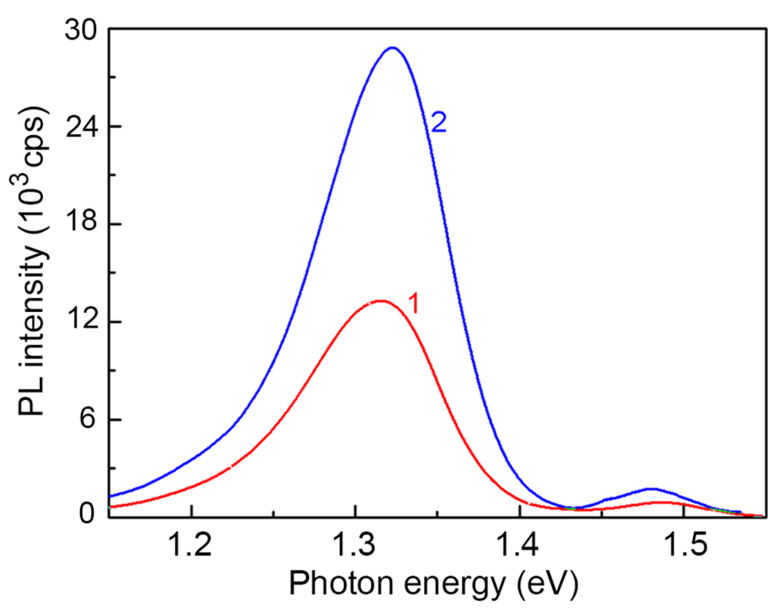

Figure 5: PL spectra of bulk (curve 1) and anodized (curve 2) GaAs samples measured at a temperature of $10 \mathrm{~K}$. anodized GaAs sample is indicative of an effective passivation during anodization of the huge internal surface of the porous sample [18].

The preservation of the quality of the GaAs compound after anodization is also confirmed by the results of XRD analysis (Figure 6). The high quality of the material produced by anodization is indicated by narrow reflexes with a full width at half maximum (FWHM) of about $0.08^{\circ}$. The predominance of (111) and (333) reflexes in the XRD pattern indicates also to the preservation of the initial (111)B crystallographic orientation of the sample.

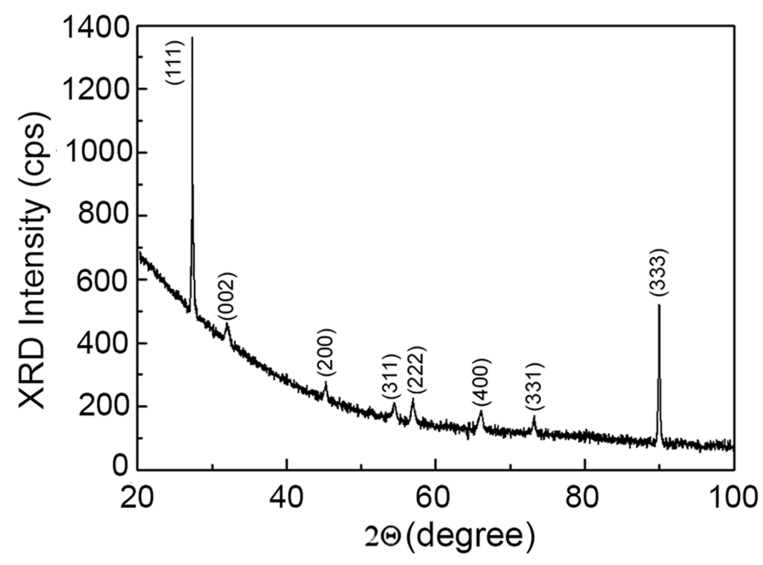

Figure 6: XRD pattern of the anodized $\mathrm{GaAs}(111) \mathrm{B}$ sample.

To demonstrate the applicability of the nanowires for device fabrication, a photodetector for the IR region of the spectrum was tested, as described in the Experimental section. A special design of contacts was applied via laser beam lithography on selected nanowires. As illustrated in Figure 7A, defined regions (bright regions) were opened in the photoresist (dark regions) for further metal deposition. Note that the nanowire was visible in the optical microscope due to its length of $70 \mu \mathrm{m}$, despite the small diameter. The distance between the contacts is $20 \mu \mathrm{m}$. A photograph of five contacted nanowires on a glass substrate after $\mathrm{Cr} / \mathrm{Au}$ deposition and lift-off is presented in the inset of the Figure 7A. The photocurrent build-up and relaxation for a photodetector produced on a nanowire with a diameter of $400 \mathrm{~nm}$ is presented in Figure 7B for an IR illumination density of $800 \mathrm{~mW} \cdot \mathrm{cm}^{-2}$. One can see that the current increases by a factor of four in magnitude under illumination with IR light.

The current-voltage characteristics measured with and without illumination reveal a good ohmic quality of the prepared $\mathrm{Cr} / \mathrm{Au}$ contacts (Figure 8). Therefore, one can conclude that the detector works in the photoconductor mode. 

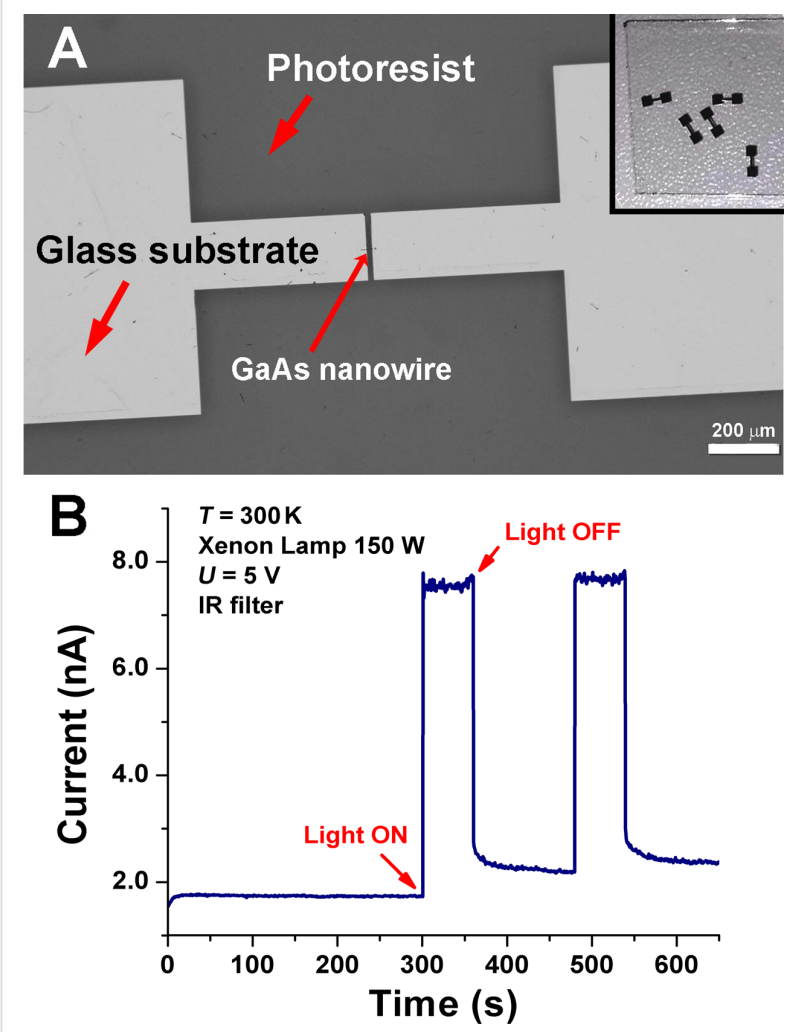

Figure 7: (A) Optical microscopy image of the opened regions in the photoresist on the glass substrate for deposition of the metal contacts on the selected $\mathrm{GaAs}$ nanowire. The inset in $(A)$ shows a photo of five contacted GaAs nanowires on the same glass substrate. (B) Photocurrent build-up and relaxation of the photodetector measured for an IR illumination density of $800 \mathrm{~mW} \cdot \mathrm{cm}^{-2}$.

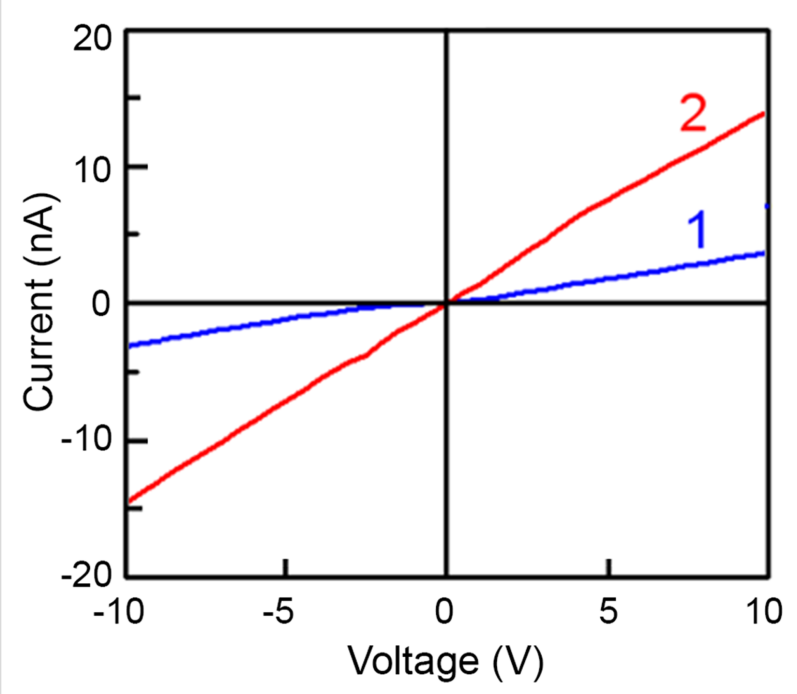

Figure 8: Current-voltage characteristics measured in dark (curve 1) and under IR illumination with power density of $800 \mathrm{~mW} \cdot \mathrm{cm}^{-2}$ (curve 2) for the GaAs nanowire photodetector with the design shown in Figure 7.
The responsivity of the detector is defined as

$$
R=\frac{I_{\text {photo }}-I_{\text {dark }}}{P_{\text {ill }}},
$$

where $I_{\text {photo }}$ is the photocurrent of the photodetector, $I_{\text {dark }}$ is the dark current, and $P_{\text {ill }}$ is the illumination power on the photodetector. The calculated responsivity of the GaAs nanowire photodetector equals $100 \mathrm{~mA} \cdot \mathrm{W}^{-1}$, according to the data presented in Figure 7B for a nanowire with a diameter of $400 \mathrm{~nm}$ and a length of $20 \mu \mathrm{m}$ biased at $5 \mathrm{~V}$.

The estimation of the detectivity $\mathrm{D}^{*}$, which is defined as

$$
D^{*}=\frac{R \sqrt{A}}{\sqrt{2 e I_{\mathrm{dark}}}},
$$

where $A$ is the active area of the photodetector, and $e$ is the elementary charge, gives a value of ca. $1.2 \times 10^{9} \mathrm{~cm} \cdot \mathrm{Hz}^{1 / 2} \cdot \mathrm{W}^{-1}$, under the assumption that shot noise is the primary source of noise in the detector [32].

Taking into account that the photodetector works in the photoconductor mode, the photocurrent increases linearly with increasing bias. This results in increasing responsivity and detectivity with increasing bias. For instance, the responsivity increases by a factor of three after increasing the bias from 5 to $20 \mathrm{~V}$. The measured parameters vary among the five investigated devices basically due to the different diameters of the nanowires. The responsivity measured at the same excitation power density of $800 \mathrm{~mW} \cdot \mathrm{cm}^{-2}$ at a bias of $5 \mathrm{~V}$ decreases with decreasing nanowire diameter. The detectivity also decreases with decreasing diameter, but to a lesser extent.

The obtained values of responsivity and detectivity are comparable with those previously reported for a graphene/GaAs NW photodetector with a Schottky junction working at $532 \mathrm{~nm}$ radiation [33]. The detectivity of our photodetector is better than that reported for a GaAsSb NW IR detector $(1300 \mathrm{~nm})$, although the responsivity of the GaAsSb NW detector is better [34]. A photodetector based on a single GaAs nanowire with a responsivity of $1.2 \mathrm{~mA} \cdot \mathrm{W}^{-1}$ has been recently reported on a nanowire prepared by chemical beam epitaxy (CBE) with a vapor-liquid-solid (VLS) growth procedure [35]. This value is by two orders of magnitude lower than the responsivity of our photodetector. However, one should take into account that it was fabricated with a nanowire that is one order of magnitude thinner than our nanowire prepared by electrochemical etching. The detectivity of our GaAs nanowire detector working in the 
photoconductor mode is by a factor of 1.5 better than the value obtained recently on molecular beam epitaxy (MBE)-grown $\mathrm{Si}$-doped GaAs nanowires with a carrier concentration of $1.47 \times 10^{17} \mathrm{~cm}^{-3}$, working in the field-effect transistor (FET) mode at similar excitation power densities (around $800 \mathrm{~mW} \cdot \mathrm{cm}^{-2}$ ) but with radiation of $532 \mathrm{~nm}$ wavelength [36]. At the same time, the authors of [36] succeeded to improve the detectivity of the NW FET detector by one order of magnitude and also to attain record responsivities of the order of $1 \mathrm{kA} \cdot \mathrm{W}^{-1}$ after optimization of the carrier concentration in the GaAs NWs and of the photodetector design. We suppose that the parameters of IR photodetectors based on nanowires prepared by anodization can also be significantly improved after corresponding optimization.

A drawback of photoconductive detectors based on semiconductor nanowires is related to their long-relaxation phenomena caused by the strong surface band bending effects [37]. In contrast, much shorter relaxation times are inherent to photodetectors based on interdigitated metal-semiconductor-metal structures with Schottky diodes. However, a very low feature size is needed for such structures, which makes photolithography challenging [38].

\section{Conclusion}

This study demonstrates possibilities to produce porous GaAs structures with a controlled degree of porosity through the anodization of GaAs(111) wafers in a neutral, environmentally friendly $\mathrm{NaCl}$ electrolyte. Porous morphologies with pores oriented perpendicularly to the wafer surface are obtained through potentiostatic anodization of GaAs(111)B surfaces at low applied potentials. With increasing the applied potential in the potentiostatic anodization mode, or the current in the galvanostatic anodizing mode, a higher number of tilted pores are produced in addition to those oriented perpendicularly to the wafer surface. When the anodization is performed on the $\mathrm{GaAs}(111) \mathrm{A}$ surface, a porous morphology with crossing pores is obtained, and the degree of porosity increases with increasing the anodizing current. Since these results are similar to those previously observed after anodization of GaAs wafers in $\mathrm{HCl}$ electrolytes, one can conclude that the etching behavior is mainly determined by the type of anions. No nanowires are produced under any anodizing conditions in $\mathrm{NaCl}$ electrolyte. On the other hand, high-aspect-ratio triangular shape GaAs nanowires are obtained by anodizing in a $\mathrm{HNO}_{3}$ electrolyte at an applied potential of $3 \mathrm{~V}$, and the uniformity and orientation of these nanowire arrays are much better than those produced previously with anodizing in alkaline $\mathrm{KOH}$ electrolytes. The produced GaAs nanowires prove to be suitable for the development of IR photodetectors with good sensitivity and dynamic characteristics.

\section{Experimental}

Electrochemical anodization. Crystalline $500 \mu \mathrm{m}$ thick (111)oriented substrates of $\mathrm{Si}$-doped $\mathrm{n}$-GaAs with a free electron concentration of $2 \times 10^{18} \mathrm{~cm}^{-3}$ were used in this study. The samples were sonicated in acetone for $15 \mathrm{~min}$, cleaned in distilled water and dried. In order to remove the native oxide from the surface, the samples were dipped in a $\mathrm{HCl} / \mathrm{H}_{2} \mathrm{O}(1: 3)$ solution for $2 \mathrm{~min}$. The electrical contacts to the sample were prepared with silver paste, then the samples were pressed against an O-ring in a Teflon cell with the $0.2 \mathrm{~cm}^{2}$ area exposed to the electrolyte. The electrolytes used in this study were $1.75 \mathrm{M} \mathrm{NaCl}$ and $1 \mathrm{M} \mathrm{HNO}_{3}$. The experiments were performed in a three-electrode configuration, with a Pt mesh with a surface area of $6 \mathrm{~cm}^{2}$ acting as counter electrode, a saturated $\mathrm{Ag} / \mathrm{AgCl}$ reference electrode and the sample as working electrode. The anodization was performed in galvanostatic as well as potentiostatic regimes at room temperature $\left(T=23{ }^{\circ} \mathrm{C}\right)$. Analysis of morphology and chemical composition of the anodized GaAs crystals was carried out using scanning electron microscopy (Zeiss Sigma and TESCAN Vega TS 5130 MM equipped with an Oxford Instruments INCA Energy EDX system operated at $20 \mathrm{kV}$ ). The photoluminescence spectra were measured with a double spectrometer with resolution better than $1 \mathrm{meV}$ under excitation by the $514 \mathrm{~nm}$ line of an $\mathrm{Ar}^{+}$ SpectraPhysics laser. The samples were mounted on the cold station of a LTS-22-C-330 cryogenic system. X-ray diffraction analysis of the samples was performed with a Philips X-Pert MPD System with $\mathrm{Cu} \mathrm{K} \alpha_{1}$ radiation.

Electrical contacts to GaAs nanowires. The contacts were realized using laser leam lithography ( $\mu$ PG 101, Heidelberg Instruments). After the formation of nanowires via anodization, the GaAs substrates were treated in an ultrasound bath for $15 \mathrm{~s}$ in ethanol. Subsequently, a few drops of the ethanol suspension containing nanowires were deposited on a glass substrate followed by a gentle blow drying to remove the ethanol. A doublelayer resist (LOR 3B and ma-P 1205) was spin-coated on the glass substrate with the GaAs nanowires and was exposed with the pattern containing the contact pad structure of $1.5 \mathrm{~mm} \times 1.5 \mathrm{~mm}$ using the laser writer. After the development of the exposed contact pad structure, a thin layer of $50 \mathrm{~nm} \mathrm{Cr}$ followed by $250 \mathrm{~nm}$ Au layer was sputtered using a magnetron from Torr International Inc model No: CRC622-2G2-RF-DC and lift-off was performed with Microposit remover 1165 at $50{ }^{\circ} \mathrm{C}$.

Photoelectrical characterization. To excite photoconductivity in the GaAs nanowires, the radiation from a Xenon lamp DKSS-150 was used. An optical filter was used to select radiation from the near-IR spectral range (700-2500 nm, optical power $130 \mathrm{~mW}$ ). The current through the samples was 
measured by means of a Keithley's Series 2400 source measure unit. Since the photoconductivity decay time is long enough, a mechanical shutter was used in the relaxation experiments. The signal from the source measure unit was fed to computer via IEEE-488 interface for further data processing. The measurements were performed at $300 \mathrm{~K}$.

\section{Acknowledgements}

The manuscript contains contributions from all authors. All authors have given approval to the final version of the manuscript.

\section{Funding}

Eduard Monaico thanks the Alexander von Humboldt Foundation for support. The authors acknowledge financial support from the Ministry of Education, Culture and Research of Moldova under the Grant \#20.80009.5007.20. This work has received partial funding from the bilateral Moldova-Belarus Program under the grant 19.80013.50.07.03A/BL as well as from the European Commission under the Grant \#810652 "NanoMedTwin".

\section{ORCID ${ }^{\circledR}$ iDs}

Elena I. Monaico - https://orcid.org/0000-0002-9486-2589 Eduard V. Monaico - https://orcid.org/0000-0003-3293-8645 Veaceslav V. Ursaki - https://orcid.org/0000-0003-4488-850X Vitalie Postolache - https://orcid.org/0000-0002-0391-1526 Karin Leistner - https://orcid.org/0000-0002-8049-4877 Kornelius Nielsch - https://orcid.org/0000-0003-2271-7726 Ion M. Tiginyanu - https://orcid.org/0000-0003-0893-0854

\section{References}

1. Föll, H.; Langa, S.; Carstensen, J.; Christophersen, M.; Tiginyanu, I. M. Adv. Mater. (Weinheim, Ger.) 2003, 15, 183-198. doi:10.1002/adma.200390043

2. Föll, H.; Carstensen, J.; Frey, S. J. Nanomater. 2006, 91635. doi:10.1155/jnm/2006/91635

3. Kochergin, V.; Föll, H. Porous Semiconductors; Springer, 2009 doi:10.1007/978-1-84882-578-9

4. Tiginyanu, I.; Monaico, E.; Monaico, E. Electrochem. Commun. 2008, 10, 731-734. doi:10.1016/j.elecom.2008.02.029

5. Tiginyanu, I. M.; Ursaki, V. V.; Monaico, E.; Enachi, M.; Sergentu, V. V.; Colibaba, G.; Nedeoglo, D. D.; Cojocaru, A.; Föll, H. J. Nanoelectron. Optoelectron. 2011, 6, 463-472. doi:10.1166/jno.2011.1197

6. Bian, Y.; Zheng, Z.; Zhao, X.; Liu, L.; Su, Y.; Xiao, J.; Liu, J.; Zhu, J.; Zhou, T. J. Lightwave Technol. 2013, 31, 1973-1979. doi:10.1109/jt.2013.2263217

7. Wallentin, J.; Anttu, N.; Asoli, D.; Huffman, M.; Åberg, I.; Magnusson, M. H.; Siefer, G.; Fuss-Kailuweit, P.; Dimroth, F.; Witzigmann, B.; Xu, H. Q.; Samuelson, L.; Deppert, K.; Borgström, M. T. Science 2013, 339, 1057-1060. doi:10.1126/science.1230969
8. Service, R. F. Science 2013, 339, 263. doi:10.1126/science.339.6117.263

9. Cui, Y.; Wang, J.; Plissard, S. R.; Cavalli, A.; Vu, T. T. T.; van Veldhoven, R. P. J.; Gao, L.; Trainor, M.; Verheijen, M. A.; Haverkort, J. E. M.; Bakkers, E. P. A. M. Nano Lett. 2013, 13, 4113-4117. doi:10.1021/nl4016182

10. Czaban, J. A.; Thompson, D. A.; LaPierre, R. R. Nano Lett. 2009, 9, 148-154. doi:10.1021/nl802700u

11. Wang, J.; Gudiksen, M. S.; Duan, X.; Cui, Y.; Lieber, C. M. Science 2001, 293, 1455-1457. doi:10.1126/science.1062340

12. Duan, X.; Huang, Y.; Cui, Y.; Wang, J.; Lieber, C. M. Nature 2001, 409 66-69. doi:10.1038/35051047

13. Tomioka, K.; Yoshimura, M.; Fukui, T. Nature 2012, 488, 189-192. doi:10.1038/nature11293

14. Monaico, E.; Tiginyanu, I.; Volciuc, O.; Mehrtens, T.; Rosenauer, A.; Gutowski, J.; Nielsch, K. Electrochem. Commun. 2014, 47, 29-32. doi:10.1016/j.elecom.2014.07.015

15. Asoh, H.; Kotaka, S.; Ono, S. Electrochem. Commun. 2011, 13, 458-461. doi:10.1016/j.elecom.2011.02.020

16. Ono, S.; Kotaka, S.; Asoh, H. Electrochim. Acta 2013, 110, 393-401. doi:10.1016/j.electacta.2013.06.025

17. Li, X.; Guo, Z.; Xiao, Y.; Um, H. D.; Lee, J. H. Electrochim. Acta 2011, 56, 5071-5079. doi:10.1016/j.electacta.2011.03.084

18. Tiginyanu, I. M.; Ursaki, V. V.; Monaico, E.; Foca, E.; Föll, H. Electrochem. Solid-State Lett. 2007, 10, D127-D129. doi:10.1149/1.2771076

19. Volciuc, O.; Monaico, E.; Enachi, M.; Ursaki, V. V.; Pavlidis, D.; Popa, V.; Tiginyanu, I. M. Appl. Surf. Sci. 2010, 257, 827-831. doi:10.1016/j.apsusc.2010.07.074

20. Schwab, M. J.; Han, J.; Pfefferle, L. D. Appl. Phys. Lett. 2015, 106, 241603. doi:10.1063/1.4922702

21. Monaico, E.; Moise, C.; Mihai, G.; Ursaki, V. V.; Leistner, K.; Tiginyanu, I. M.; Enachescu, M.; Nielsch, K. J. Electrochem. Soc. 2019, 166, H3159-H3166. doi:10.1149/2.0251905jes

22. Zhang, C.; Yuan, G.; Bruch, A.; Xiong, K.; Tang, H. X.; Han, J. J. Electrochem. Soc. 2018, 165, E513-E520. doi:10.1149/2.1181810jes

23. Langa, S.; Carstensen, J.; Christophersen, M.; Steen, K.; Frey, S.; Tiginyanu, I. M.; Föll, H. J. Electrochem. Soc. 2005, 152, C525-C531. doi:10.1149/1.1940847

24. Prunici, P.; Irmer, G.; Monecke, J.; Sirbu, L.; Tiginyanu, I. Phys. Status Solidi A 2005, 202, 1562-1566. doi:10.1002/pssa.200461183

25. DeJarld, M.; Shin, J. C.; Chern, W.; Chanda, D.; Balasundaram, K.; Rogers, J. A.; Li, X. Nano Lett. 2011, 11, 5259-5263. doi:10.1021/nl202708d

26. Song, Y.; Oh, J. J. Mater. Chem. A 2014, 2, 20481-20485. doi:10.1039/c4ta05095a

27. Asoh, H.; Kotaka, S.; Ono, S. Mater. Res. Express 2014, 1, 045002. doi:10.1088/2053-1591/1/4/045002

28. Capizzi, M.; Emiliani, V.; Frova, A.; Sarto, F. Phys. Rev. B 1993, 47, 4301-4306. doi:10.1103/physrevb.47.4301

29. Sauncy, T.; Palsule, C. P.; Holtz, M.; Gangopadhyay, S.; Massie, S. Phys. Rev. B 1996, 53, 1900-1906. doi:10.1103/physrevb.53.1900

30. Yu, P. W.; Fischer, D. W.; Sizelove, J. R. Semicond. Sci. Technol. 1992, 7, 556-561. doi:10.1088/0268-1242/7/4/020

31. Deepak; Lakshminarayana, N. Bull. Mater. Sci. 2001, 24, 225-229. doi:10.1007/bf02710106 
32. Sirkeli, V. P.; Yilmazoglu, O.; Hajo, A. S.; Nedeoglo, N. D.; Nedeoglo, D. D.; Preu, S.; Küppers, F.; Hartnagel, H. L. Phys. Status Solidi RRL 2018, 12, 1700418. doi:10.1002/pssr.201700418

33. Luo, Y.; Yan, X.; Zhang, J.; Li, B.; Wu, Y.; Lu, Q.; Jin, C.; Zhang, X.; Ren, X. Nanoscale 2018, 10, 9212-9217. doi:10.1039/c8nr00158h

34. Li, Z.; Yuan, X.; Fu, L.; Peng, K.; Wang, F.; Fu, X.; Caroff, P.; White, T. P.; Hoe Tan, H.; Jagadish, C. Nanotechnology 2015, 26, 445202. doi:10.1088/0957-4484/26/44/445202

35. García Núñez, C.; Braña, A. F.; López, N.; Pau, J. L.; García, B. J. Nanotechnology 2020, 31, 225604. doi:10.1088/1361-6528/ab76ee

36. Chen, X.; Wang, D.; Wang, T.; Yang, Z.; Zou, X.; Wang, P.; Luo, W.; Li, Q.; Liao, L.; Hu, W.; Wei, Z. ACS Appl. Mater. Interfaces 2019, 11 , 33188-33193. doi:10.1021/acsami.9b07891

37. Chen, H.-Y.; Chen, R.-S.; Rajan, N. K.; Chang, F.-C.; Chen, L.-C.; Chen, K.-H.; Yang, Y.-J.; Reed, M. A. Phys. Rev. B 2011, 84, 205443. doi:10.1103/physrevb.84.205443

38. Berger, P. R. Proc. SPIE 2001, 4285, 198-207. doi:10.1117/12.426888

\section{License and Terms}

This is an Open Access article under the terms of the Creative Commons Attribution License (http://creativecommons.org/licenses/by/4.0). Please note that the reuse, redistribution and reproduction in particular requires that the authors and source are credited.

The license is subject to the Beilstein Journal of Nanotechnology terms and conditions: (https://www.beilstein-journals.org/bjnano)

The definitive version of this article is the electronic one which can be found at: doi:10.3762/bjnano.11.81 